\title{
AN EXTENSION OF HAYNSWORTH'S DETERMINANT INEQUALITY
}

\author{
D. J. HARTFIEL
}

Abstract. Let $A$ and $B$ be positive definite hermitian matrices of order $n$. This paper improves a lower bound given for $|A+B|$ by E. V. Haynsworth.

In [1] E. V. Haynsworth proves that if $A$ and $B$ are positive definite hermitian matrices of order $n$ then

$$
|A+B| \geqq\left(1+\sum_{i=1}^{n-1} \frac{\left|B_{i}\right|}{\left|A_{i}\right|}\right)|A|+\left(1+\sum_{i=1}^{n-1} \frac{\left|A_{i}\right|}{\left|B_{i}\right|}\right)|B|
$$

where $A_{i}, B_{i}$ denote the principal submatrices of order $i$ in the upper left corner of $A$ and $B$ respectively. This note uses the same techniques developed in [1] to extend the inequality. This is done by applying the following elementary calculus result.

If $f(x)=a x+b x^{-1}$ with $a$ and $b$ positive, then $\min _{0<x<\infty} f(x)$ is achieved at $(b / a)^{1 / 2}$ with $f\left((b / a)^{1 / 2}\right)=2(a b)^{1 / 2}$.

We also use the following lemma in the argument of the extended result.

LEMMA $^{1}$ [1, LEMMA 2, p. 514]. If $A$ and $B$ are positive definite hermitian matrices of order $n$ then

$$
\left|(A+B) /\left(A_{n-1}+B_{n-1}\right)\right| \geqq|A| /\left|A_{n-1}\right|+|B| /\left|B_{n-1}\right| .
$$

The extended inequality is as follows.

THEOREM. Suppose $A$ and $B$ are positive definite hermitian matrices of order $n$, then

$|A+B| \geqq\left(1+\sum_{i=1}^{n-1} \frac{\left|B_{i}\right|}{\left|A_{i}\right|}\right)|A|+\left(1+\sum_{i=1}^{n-1} \frac{\left|A_{i}\right|}{\left|B_{i}\right|}\right)|B|+\left(2^{n}-2 n\right)(|A||B|)^{1 / 2}$.

Received by the editors November 6, 1972 .

AMS (MOS) subject classifications (1970). Primary 15A15; Secondary 15A57, $15 \mathrm{~A} 45$.

Key words and phrases. Determinant, hermitian, inequality.

${ }^{1}$ We use the notation $X / Y$ instead of $X Y^{-1}$ to be consistent with the source. 
Proof. The proof of the theorem is by induction on the order $k$ of $A$ and $B$. For $k=1$ the inequality reduces to $|A+B| \geqq|A|+|B|$ which is clearly true. Suppose therefore that the inequality holds for all pairs of positive definite hermitian matrices $A$ and $B$ of order $k$ with $k<n$. Now suppose $A$ and $B$ are positive definite hermitian matrices of order $n$. Then, by applying the results of the lemma,

$$
\begin{aligned}
|A+B| & =\left|A_{n-1}+B_{n-1}\right|\left|(A+B) /\left(A_{n-1}+B_{n-1}\right)\right| \\
& \geqq\left|A_{n-1}+B_{n-1}\right|\left[|A| /\left|A_{n-1}\right|+|B| /\left|B_{n-1}\right|\right]
\end{aligned}
$$

and hence, by the induction hypothesis,

$$
\begin{aligned}
|A+B| \geqq & {\left[\left(1+\sum_{i=1}^{n-2} \frac{\left|B_{i}\right|}{\left|A_{i}\right|}\right)\left|A_{n-1}\right|\right.} \\
& +\left(1+\sum_{i=1}^{n-2} \frac{\left|A_{i}\right|}{\left|B_{i}\right|}\right)\left|B_{n-1}\right| \\
& \left.+\left[2^{n-1}-2(n-1)\right]\left(\left|A_{n-1}\right|\left|B_{n-1}\right|\right)^{1 / 2}\right]\left[\frac{|A|}{\left|A_{n-1}\right|}+\frac{|B|}{\left|B_{n-1}\right|}\right] \\
= & \left.+\sum_{i=1}^{n-2} \frac{\left|B_{i}\right|}{\left|A_{i}\right|}\right)|A|+\left(1+\sum_{i=1}^{n-2} \frac{\left|A_{i}\right|}{\left|B_{i}\right|}\right)|B| \\
& +\left(1+\sum_{i=1}^{n-2} \frac{\left|B_{i}\right|}{\left|A_{i}\right|}\right) \frac{\left|A_{n-1}\right|}{\left|B_{n-1}\right|}|B|+\left(1+\sum_{i=1}^{n-2} \frac{\left|A_{i}\right|}{\left|B_{i}\right|} \frac{\left|B_{n-1}\right|}{\left|A_{n-1}\right|}|A|\right. \\
& +\left[2^{n-1}-2(n-1)\right]\left(\left|A_{n-1}\right|\left|B_{n-1}\right|\right)^{1 / 2}\left[\frac{|A|}{\left|A_{n-1}\right|}+\frac{|B|}{\left|B_{n-1}\right|}\right] .
\end{aligned}
$$

Thus by combining the first and part of the third expressions, the second and part of the fourth expressions we have

$$
\begin{aligned}
|A+B| \geqq & {\left[1+\sum_{i=1}^{n-1} \frac{\left|B_{i}\right|}{\left|A_{i}\right|}\right]|A|+\left[1+\sum_{i=1}^{n-1} \frac{\left|A_{i}\right|}{\left|B_{i}\right|}\right]|B| } \\
& +\sum_{i=1}^{n-2} \frac{\left|B_{i}\right|}{\left|A_{i}\right|} \frac{\left|A_{n-1}\right||B|}{\left|B_{n-1}\right|}+\sum_{i=1}^{n-2} \frac{\left|A_{i}\right|}{\left|B_{i}\right|} \frac{\left|B_{n-1}\right||A|}{\left|A_{n-1}\right|} \\
& +\left[2^{n-1}-2(n-1)\right]\left(\left|A_{n-1}\right|\left|B_{n-1}\right|\right)^{1 / 2}\left[\frac{|A|}{\left|A_{n-1}\right|}+\frac{|B|}{\left|B_{n-1}\right|}\right] ;
\end{aligned}
$$

hence by applying the calculus result termwise to a pair of corresponding 
terms in expressions three and four and also to expression five we have

$$
\begin{aligned}
|A+B| \geqq & \left(1+\sum_{i=1}^{n-1} \frac{\left|B_{i}\right|}{\left|A_{i}\right|}\right)|A|+\left(1+\sum_{i=1}^{n-1} \frac{\left|A_{i}\right|}{\left|B_{i}\right|}\right)|B|+\sum_{i=1}^{n-2} 2(|A||B|)^{1 / 2} \\
& +\left[2^{n-1}-2(n-1)\right]\left(\left|A_{n-1}\right|\left|B_{n-1}\right|\right)^{1 / 2}\left[2\left(|A||B| /\left|A_{n-1}\right|\left|B_{n-1}\right|\right)^{1 / 2}\right] \\
\geqq & \left(1+\sum_{i=1}^{n-1} \frac{\left|B_{i}\right|}{\left|A_{i}\right|}\right)|A|+\left(1+\sum_{i=1}^{n-1} \frac{\left|A_{i}\right|}{\left|B_{i}\right|}\right)|B|+\left(2^{n}-2 n\right)(|A||B|)^{1 / 2}
\end{aligned}
$$

This is the conclusion of the Theorem.

As a direct corollary we have the following interesting inequality.

Corollary. If $A$ and $B$ are positive definite hermitian matrices of order $n$ then $|A+B| \geqq|A|+|B|+\left(2^{n}-2\right)(|A||B|)^{1 / 2}$.

Proof. By Theorem 1,

$$
\begin{aligned}
|A+B| \geqq & |A|+|B|+\left(\sum_{i=1}^{n-1} \frac{\left|B_{i}\right|}{\left|A_{i}\right|}\right)|A|+\left(\sum_{i=1}^{n-1} \frac{\left|A_{i}\right|}{\left|B_{i}\right|}\right)|B| \\
& +\left(2^{n}-2 n\right)(|A||B|)^{1 / 2}
\end{aligned}
$$

and so, by applying the calculus result on the third and fourth expressions,

$$
\begin{aligned}
|A+B| & \geqq|A|+|B|+2(n-1)(|A||B|)^{1 / 2}+\left(2^{n}-2 n\right)(|A||B|)^{1 / 2} \\
& =|A|+|B|+\left(2^{n}-\cdot 2\right)(|A||B|)^{1 / 2} .
\end{aligned}
$$

It is, of course, easily seen by continuity that the Corollary also holds for positive semidefinite hermitian matrices as well. Another feature of both results is that equality holds when $A=B$.

\section{REFERENCE}

1. E. V. Haynsworth, Applications of an inequality for the Shur complement, Proc. Amer. Math. Soc. 24 (1970), 512-516. MR 41 \#241.

Mathematics Department, Texas A\&M University, College Station, Texas 77843 